\begin{tabular}{lll}
\hline Volume 1 & Number 7 & 14 SEPTEMBER 2016 \\
\hline
\end{tabular}

GILBERT T. GONZALEZ

\title{
DIVIDE OR CONVERGE? ${ }^{1}$
}

\section{A major change in strategy: was it time to become a divisional organization?}

Gilbert Gonzalez, founder and CEO of Mission Critical Solutions (MCS), was reflecting on the path forward for his company. MCS maintained and updated a five-year strategic plan each year since its inception in 1990. In the early years, the strategic plan focused on the key strategic factors (KSFs) and resulted in a narrow mission. Year after year, that focus on the KSFs was rewarded by the market. Doing the most important things well kept the organization focused and efficient.

The consistently evolving mission of the organization necessitated adding new products, services, and solutions. In the early 1990s, deploying PCs, implementing local area networks (LANs), and providing onsite support met the challenges their customers faced. A few years later, the company's clients' needs led to the addition of a Wide Area Network (WAN) solution to aggregate offices and link applications. By 1996, information technologies (IT) and telecommunications began to converge. Common structured cable plants and digital trunks sharing voice and data traffic were new and mandatory elements of the clients' solutions. Subtly, the technologies evolved and converged. Internet Protocol (IP) Telephony and Unified Communications converged onto the clients' LAN/WAN networks. It was clear that most technology would speak IP in the future. In the early 2000s, building automation, audio video (AV), security, surveillance, and control system endpoints converged onto the network. The trend of unusual devices converging onto the network, also known as "the Internet of things," will continue. Wireless innovations and mobility meant that technology could move not just around the facility, but also around the world.

For MCS, the end result of this phenomenon was a group of specialized departments and teams: information technologies, passive cabling systems, electrical systems, building automation and controls, audio video, security and surveillance, and unified communications. All of these departments, staffed 200 associates strong, were coordinated by the project management team, and collaborated to meet the requirements of the company's clients. Thus, the marketing elevator speech for MCS required a tall building! Their clients expressed surprise and confusion at the breadth of services offered. While the devices had converged, the clients' perception of their vendors and providers had not. The question at hand was whether MCS should stay converged in a functional organization strategy with capability-based teams, or organize into separate standalone entities with unique identities and separate overheads, focusing more on the individual capability-based technology specialization.

\footnotetext{
${ }^{1}$ Copyright (C) 2016, Gilbert T. Gonzalez. This case was prepared for the purpose of class discussion, and not to illustrate the effective or ineffective handling of an administrative situation. Names and some information have been disguised. This case is published under a Creative Commons BY-NC license. Permission is granted to copy and distribute this case for non-commercial purposes, in both printed and electronic formats.
} 


\section{Company History}

Gilbert Gonzalez, a new graduate of the University of South Florida’s MBA program, founded MCS in 1989. While completing his graduate work, Gonzalez discovered his passion for small business automation. The advent of the personal computer and networking timed perfectly with his life course. With little capital, he built a small practice selling microcomputer network services to the small and medium-sized business market. The services practice fit perfectly with his passion for technology and the limited working capital of his new small startup.

From 1989 to 1992, the company established its roots providing fee-based services to the commercial markets implementing information technology systems. As network standardization evolved, so did the need for Structured Cabling Systems (SCS). Standards based wiring schemes were necessary to support the growth in microcomputer based networked workstations, shared printers, and file servers. MCS began providing the design and installation of the SCS systems, building infrastructures to support the evolving Local Area Network (LAN). This capability was in high demand and grew quickly. In 1997, MCS made the decision to broaden their markets served, adding local government and school districts.

The advent of SCS and Telephony technologies had MCS supporting the convergence of traditional telecommunication systems to the IP network. Unified communications was in its infancy, and MCS was there to help their clients apply this new application to their network. Both the local and Wide Area Networks (WAN) were being deployed with great success.

As the year 2000 approached, the Y2K scare disrupted the focus on advancing the implementation of technologies, and forced customers to spend capital expenditure dollars on testing and addressing concerns of hard-coded software that did not adapt to the date change. Ensuring the date format would not disrupt their network systems was imperative to day-to-day operations. Rather than chase this short-term niche of software testing and correcting, MCS chose to turn their attention to broadening their reach to the federal government. The shortage in qualified small businesses that were willing and able to service the federal customer was significant, and MCS's strategy was rewarded with new clients and satisfying growth.

Then, having implemented a sales and marketing program that covered commercial, state, local, education (SLED), and federal clients, MCS focused on adding new capabilities that were converging onto their customers' IP networks. Numerous building automation endpoints, security and life safety endpoints, and audio and video media endpoints converged onto the clients' networks. MCS was there to show clients how to cause these disparate systems to attach to the network, and collaborate in ways that saved time and money. Through 2014, MCS continued to add the products, skills, and experience needed to support the six current capabilities that had converged onto the IP based network.

\section{MCS's Mission}

The organization's mission was to be an IP Converged Technology solutions provider with a national footprint. From its inception, the strategic mission statement of MCS had evolved into: "To provide reliable, competitively priced, and superior mission critical IP-converged technology solutions to federal, state, and local governments, educational institutions, and commercial markets primarily in the continental United States (CONUS) and strategically outside the continental Unites States (OCONUS) as well as in forward support of Department of Defense (DoD) client initiatives within [their] six categories of capabilities” (Gonzalez, 2014). 


\section{MCS Capabilities}

\section{MCS's Markets and Products}

IP Converged products and services were products and systems that integrate and collaborate on a common network. MCS brought new endpoints, which collaborate in order to improve efficiency, quality, and responsiveness in the process of convergence, onto the network.

The products and services MCS designed, installed, and maintained on behalf of their clients were organized by capability. Mission Critical Solutions brought proven experience through their six major competencies (see Exhibit 4).

$>$ Information Technology

$>$ Structured Cabling

$>$ Unified Communications

$>$ Audio Visual Solutions

$>$ Security \& Life Safety

$>$ Electrical Distribution \& Specialty Contracting

\section{MCS Geographic Service Area}

Headquartered in Tampa, Florida, MCS established a broad range of Convergence Technology products and services that they grouped into three main divisions--Building Automation, Information Systems, and Telecommunications--as their core business lines. MCS's growth was consistent over 17 years, boasting a full-time staff of over 230 employees, having branch and project offices located throughout the United States, and performing successfully on several major Department of Defense (DoD) projects overseas.

With 15 Registered Communications Distribution Designers (RCDDs) on staff, more than any other small business in the country, and a résumé of many of the largest and most complex building automation projects in the Southeast United States, MCS was an industry leader in an ever-growing area of mission critical IP-Converged Technology solutions for clients nationwide. MCS held numerous hard earned telecommunications certifications such as Cisco, Avaya, Nortel, and NEC, and was a Microsoft-Certified Solutions Partner. MCS was one of only two small businesses to achieve Platinum status as a Federal Business Partner with Avaya (see Exhibit 1).

\section{Information Technology}

MCS offered a wide range of professional services to ensure the client organization was harnessing the full potential of their IT infrastructure, including assessment, design, planning, staging, configuration, and installation. They merged new technology solutions with existing IT infrastructures, upgraded critical applications, and found better ways to manage data storage and retrieval. Their IT Professional Services consultants offered the specialized experience necessary to launch and manage global IT, security, and cloud solutions effectively. MCS's service team provided tier-1 support. They used trouble ticketing and resolution logging methods to identify, document, and track required system and network modifications and repairs. MCS provided and supported servers, endpoints, and layer 3 communications needs.

MCS supported all common technologies for most manufacturers, including but not limited to:

$>$ Servers and Virtualization

$>$ Passive optical networking and Gigabit passive optical networking 
$>$ 10/100/1000BASE-T Ethernet

$>$ Licensed and unlicensed spectrum wireless and WIFI communication

$>$ Data center infrastructure and environmental equipment

$>$ Network performance monitoring

$>$ Workstations, mobile devices, tablets, printers, scanners and other endpoints

\section{Structured Cabling}

MCS designed, installed and maintained voice and data networks since 1993, when it began installing some of the first legacy LANs and WANs. The company worked with manufacturers, system integrators, and equipment suppliers to provide low cost, highly effective solutions for voice and LAN system requirements. With the advent of fiber optic technology, they aggressively trained their technicians and managers in order to first achieve and then maintain their position as a leading fiber optic cable and premise distribution contractor throughout the Southeast United States. MCS maintained this position by continuously training its staff and tackling the most complex and challenging projects. The firm provided cabling infrastructure, ranging from small data center installations to large-scale campus and base-wide backbone infrastructure.

\section{Unified Communications}

MCS provided Unified Communications and Collaboration Solutions that incorporated multiple enterprise communication technologies and applications, as well as their associated software, services and equipment. This allowed them to produce an environment that best supports the way users work, thus resulting in a workforce that was efficient anywhere and everywhere. MCS's UC group provided services including design, integration, installation, maintenance and management. They understood the need to factor in all the parts of the solution to ensure proper design from the top down. Whether they were providing messaging, conferencing, IM, mobile integration, video, or audio, all of their UC and Collaboration Solutions leveraged their consultative approach and deep technical expertise.

\section{Electrical Distribution Systems}

MCS Electrical Distribution Division's expertise included engineering, installation, proactive preventive maintenance, design, and build services. They maintained a staff specializing in the engineering, testing, and maintenance of electrical systems. Every installation, inspection, and testing was documented and quality assured by technicians who stood ready to perform any emergency repair services. MCS dedicated full-time technicians to clients whenever necessary, and provided the expertise and services to ensure the integrity of mission critical systems by understanding customer needs and operations. Their breadth of services was aimed at ensuring continuous uptime, preventing disruptive and costly outages, and providing effective data center infrastructure support. MCS developed alternative energy solutions in rural areas where grid power was not available. Through combinations of wind, solar, and generators, MCS was able to provide energy for remote endpoints.

\section{Electronic Security and Life Safety Systems}

For more than 13 years, MCS was a leader in electronic security and life safety systems installation and maintenance. They provided design and technical services to commercial, industrial, and governmental clients. The division specialized in security and life safety systems with a wide array of services. These included conceptualization, planning, design, integration, and implementation, as well as acceptance testing and follow-on support. The diversity of their experience allowed them to address a multitude of specialized areas within the fields of security and life safety systems. From perimeter intrusion systems to basic life safety, MCS could design, build, and maintain the systems. 


\section{Audio-Visual}

MCS's AV solutions group helped clients share information, meet goals, and maintain the value of audiovisual investments since 2009. They took a comprehensive approach to their audiovisual technology services, offering support for everything from product selection and design, to engineering and integration, to monitoring and managing audiovisual assets and resources. They approached AV solutions holistically, helping clients consider all the necessary components of technology decision-making and impact. Their approach took into consideration migrating AV and VTC from proprietary networks and cost centers to converge and comply with IT systems and security, while maintaining agnostic manufacturer evaluation, negotiation, and management.

\section{How Businesses Grow}

Numerous papers and studies have been done on the stages that a business goes through from inception until maturity. Most researchers agreed that there are between five and seven of those stages. One of those researchers, James Fisher, conducted a nine year study of entrepreneurial companies in the Front Range and Silicon Valley. His study, which was particularly well suited to the history of MCS, demonstrated "7 Stages of Growth" in entrepreneurial companies. The "Stages of Growth," as defined below, were based on interviews with over 700 CEOs, conducted in order to understand and decipher the patterns, behavior, and characteristics of growth in entrepreneurial enterprises (Fischer, 2006).

Table 1: The 7 Stages of Growth in Entrepreneurial Companies (Fischer, 2006).

\begin{tabular}{|c|l|l|l|l|}
\hline Stage & Theme & Staff Size & *Minimum Revenue Size & Typical Organizational Style \\
\hline I & Survival & $1-10$ & Less than 1 million dollars & Flat \\
\hline II & Ramp Up & $11-19$ & 1 million to 2 million dollars & Functional \\
\hline III & Delegation & $20-34$ & 2 million to 4 million dollars & Functional \\
\hline IV & Professional & $35-57$ & 4 million to 6 million dollars & Functional \\
\hline V & Integration & $58-95$ & 6 million to 10 million dollars & Functional or Divisional \\
\hline VI & Strategic & $96-160$ & 10 million to 20 million dollars & Divisional \\
\hline VII & Visionary & $161-500$ & 20 million and above & Divisional or Matrix \\
\hline
\end{tabular}

* Revenue size can be a poor indicator of the current stage; above revenues assume a service mission. Service firms will have significantly lower revenue than firms selling goods or bundling goods with services at any given phase.

\section{Challenges in Each Phase}

Each phase had its own unique challenges to be overcome before the business can evolve to the next stage. Gonzalez noted that MCS had in fact evolved through the first six phases and was confronting the visionary challenges of stage seven. 
It is important to recognize the common thread or theme to the challenges at each stage, as the typical business evolves. In Stage I, survival was the theme. These challenges included limited cash flow, insufficient capital, and limited labor. In this phase, there were periods of chaos and confusion. Sales growth was still the heavy focus for the founder. Sustaining sales momentum and reinvesting earnings were the keys to graduating from that phase.

In Stage II, ramping up was the theme. The key challenges included cash and capital limitations. But now hiring and leadership challenges came into the mix as well. Identifying and recruiting quality staff while driving sales growth was a heavy burden. Retaining and reinvesting earnings was still a priority in order to fuel the growth.

Delegation was the theme for Stage III. The key challenges evolved in response to the increased staff and their needs. Delegation of authority was important, and business process design was needed to provide new staff with procedures and guidelines. The founder was still highly visible, but now the enterprise was led by a middle management team. Emphasis was placed on establishing core values and corporate culture.

In Stage IV, the theme was building a professional organization. The key challenges were: developing a proper culture, improving project management skills, avoiding employee turnover, and implementing the business processes established in earlier phases. The beginnings of an executive management team had been forming. Planning and forecasting were new goals to be emphasized during the middle stage.

In Stage V, the theme was integration. Human capital was the major challenge. The onboarding of new employees was now critical to sustained quality. Growth depended on the ability to scale the staff without impacting quality. The ability for new staff to perform was paramount to sustaining sales growth. Thus, training cost was a greater burden. The process and procedures developed in early stages were tested and refined

Upon entering Stage VI, being strategic and purposeful was the theme. The key challenges continued to evolve around staff augmentation and development. Employee retention was critical to efficiency and effectively sustaining growth. Maintaining the culture with the wave of new employees was important. Process and procedures evolved as the new employee scale was obsoleting those that served in the past. A strategic plan that adapted to market change, ensured differentiation, and avoided obsolescence was the key to growth.

In Stage VII, the theme was "Visionary" and the challenges were around differentiation, profitability, loss of agility, and responding to changes in the market. In that stage, an executive management team was making key decisions based on the strategic plan in place at the time. The strategic plan was the tool that caused purposeful growth through the implementation of strategies that supported the objectives and mission established by the executive leadership. In some cases, a formal or informal board existed to assist the founder and executive team in planning (Fischer, 2006).

\section{Organizational Structures}

As firms evolved through these phases, their organizational structures must also be adapted. The organizational structure determined how the organization performed or operated. The term "organizational structure" referred to how the people in an organization are grouped and to whom they reported. Enterprises typically evolved as the number of employees increased. The startup usually began in a simple flat structure where the entrepreneur led a small team, who assisted the founder as direct reports in any task needed. In the next phase, employees were now organized by function, and typically a supervisor or manager led the function and was a direct report to the founder. Often as a direct result of 
the desire to grow, the founder might choose to add products, capabilities, or markets that had unique needs. If the enterprise was confronted with this challenge, the entrepreneur likely adapted a divisional organizational structure. Another possible reaction to growth was to implement a matrix structure. Matrix structures grouped employees by both function and product. This structure combined the best of the functional and divisional structures (Mohr, 1982).

\section{Flat Structure}

The flat structure was ordinary in micro-businesses and startups. Typically, the owner led and directed the group. Often employees were cross-trained in more than one functional area. As companies grew, they tended to become more complex and hierarchical. That caused additional staff being needed and an expanded structure, with more reporting levels and individual departments (Mohr, 1982).

\section{Functional Structure}

A functional organizational structure was a simple structure that consisted of activities such as coordination, supervision, and common task allocation. One common way of organizing people was by function. Typical functions within an organization included production, marketing, human resources, and accounting. This organizing strategy provided operational efficiency when employees became specialists within their own functional responsibility. A common problem for business's with a functional organizational structure was communication. This structure tended to discourage flexibility within the company, making the organization slower (Mohr, 1982).

\section{Divisional Structure}

The divisional structure was a product-based structure. The organization was made up of two or more self-contained divisions. A division contained all of the functions that produced the product or service. The strategy was to operate each division as a separate profit center. A key benefit of this structure was flexibility within the divisions, which created agility and speed when action was needed. One of the disadvantages of the divisional structure was that it could create unhealthy rivalries among the divisions. Another concern was the overlapping of managers, increasing the total cost for the business. Lastly, each division tended to emphasize their unique strategies over organizational goals, potentially leading to conflicting objectives (Mohr, 1982).

\section{Matrix Structure}

In a matrix structure, employees were grouped by both function and product. This structure could be the best of both worlds, creating teams that collaborated functionally and divisionally. Work teams were common when implementing a matrix organization. These teams were led by project managers and controlled the functional and divisional resources of the organization as one focused team in order to accomplish the project goals. An important feature of the matrix structure was the recognition that power was shared equally between the project manager and the functional managers. It could bring out the best performance in a complex organization, but shared power could pose many challenges and required rigorous coordination and collaboration (Mohr, 1982).

\section{Strategic Factors for 2014}

Each year, MCS updated and revised the strategic audit as a key part of the strategic planning process (Exhibit 2). The main purpose of the audit was to determine what the key strategic factors were for the 
year and to ensure that focus was placed on meeting and addressing these items. MCS placed emphasis on the following five key strategic factors for the 2015 business plan (Gonzalez, 2014).

The audit showed that MCS had a high cost of sales and a low sales efficiency ratio (revenue/sales expense) relative to its more narrow competitors. Marketing efficiency in both commercial and federal markets needed to be improved. It could be done through better prequalification, proposal creation, and closing techniques. Improving the close ratio would drive growth and improve MCS's sales efficiency ratio.

The audit further indicated that MCS's corporate culture was becoming diluted and varied by team and region. The impact of functional teams spreading across 22 states had diluted the driven and competitive culture that MCS valued. MCS needed to improve inter-company communication and to foster a single culture across the geographically dispersed teams. Team building events, temporary duty assignments, and required collaboration through work assignment would be emphasized. MCS would also begin to brand its culture through the newsletter and MCS YouTube channel.

The audit also demonstrated that MCS's current performance appraisal system was not meeting the needs of the organization, since it was hampered by blended metrics which allowed poor and moderate performers to go unnoticed. Further, it made mentoring and coaching more challenging, due to the lack of objective data available per individual. The team approach needed to be balanced with individual performance metrics. Managers would be tasked with measuring each associate with individual metrics that reflected their contributions and encouraged achievement. When necessary, additional training would be provided at the individual level.

The audit further reminded management that MCS's financial model depended on driving enough sales volume to overfund the indirect cost pools. When revenue volume fell, their fixed indirect costs were not fully funded and profits suffered. They needed to emphasize sales volume in order to overfund the indirect pool and outperform the market benchmarks.

Lastly, the audit demonstrated that MCS's key differentiator was that it could take on highly complex projects, which required many, if not all, of their converged capabilities. These projects drove revenue and supported gross profit margins. The team delivered a converged solution in less time and at lower cost than its larger business rivals. This differentiator mandated that they pursue new projects that required and rewarded MCS's willingness to take on the tough work. From Department of Defense (DOD) data centers to remote video surveillance systems across 25 miles of the border, to sophisticated tier one IT support, MCS delivered and flourished.

\section{MCS's Options}

As Gonzalez looked at the completed strategic audit for 2014 (Exhibit 3), he wondered if there was one company there or three. A look at the competition found no one firm that competed with MCS head to head in all six capabilities. Some firms offered these products and solutions, but they would oversee subcontract partners for one or more of the capabilities needed. Even large businesses, such as General Dynamics, Boeing, Harris, L3, and IBM, did not self-perform the full spectrum of capabilities MCS offered; they have all hired MCS in the past to meet their clients' needs.

Was MCS's consistent response to expanding its capabilities based on converging technologies and trends making it too broad? Could a small business continue to deliver excellence in scale and breadth? Gonzalez had recently attended a lecture by Harvard professor Francis Frie. In her book, published with Anne Morris, they suggested four truths: 


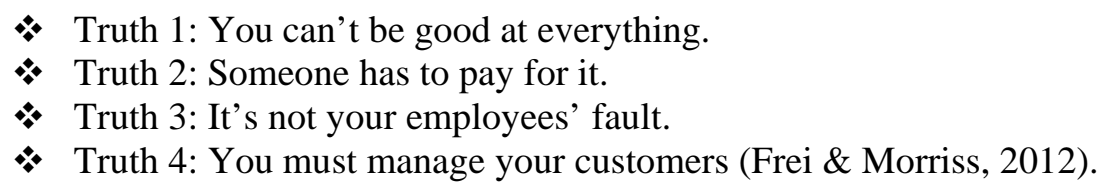

In summary, their position was that organizations suffer from "exhaustive mediocrity" (Frei \& Morriss, 2012). The stress and pressure of trying to be great at all things to all people would exhaust an organization and cause inevitable mediocrity. Gonzalez wondered if this was MCS's fate and whether their current strategy could be limiting their growth, expertise, and profitability. If this was a concern, then what were the options for the future?

The first option was to move forward as they had in the past. While growth had slowed, one could easily argue that any growth after the Great Recession, sequestration, and fiscal cliff was, in and of itself, a feat to celebrate. Further, their culture was to grow, innovate, and share new technologies. The team was built to respond to any and all technologies that converged onto the IP network.

Gonzalez also considered spinning off into three distinct and autonomous organizations, each with separate intraprenuerial leadership operating with individual profit and loss responsibility. These three new entities would roll up into a single holding company, owned and controlled by the same shareholder. In essence, MCS would reorganize by market segments that more closely aligned with typical competitors such as:

$>$ Unified Communications and IT design build services

$>$ Building automation and specialty contracting

$>$ Operations and maintenance and long term staffing

Could this make sense and, if so, over what time period would it occur? How would MCS market the complex strategic projects with three identities? What would be the impact to profits? Would the scale and mastery elements offset the cost increase of duplicate selling, general, and administrative expenses? Could MCS identify three intrapreneurial leaders to energize and lead the separate entities? Next, he considered segregating the existing organization by its role in the product life cycle with two separate entities serving the client's needs based on the position in that life cycle. This strategy involved creating two distinct entities organized by their role orientation towards creation versus maintenance. The two new entities would be:

$>$ Design and building of converged IP systems

$>$ Operations, maintenance, and long term staffing of existing converged IP systems

Lastly, he considered consolidating into one organization, and divesting of some capabilities. If MCS were to do this, then which of the six capabilities would remain? Would narrow mean better? Would mastery occur that would improve MCS's long-term success? 


\section{Pros and Cons of each Strategy}

\section{Option A: Stay the course.}

Pros

$>$ No distraction or stress from implementing organizational change.

$>$ No requirement to dilute and communicate a new set of missions, objectives, and strategies.

$>$ Preserve historical differentiator as a turnkey provider of complex converged technologies.

$>$ Sales team with six capabilities and hundreds of products and services to sell.

$>$ Shared selling, general, and administrative expenses along with a common indirect cost pool, which kept cost low.

$>$ Revenue potential and growth optimized across the three client markets and six capabilities.

\section{Cons}

$>$ Individual associates must stay current on a broad array of technologies, making mastery difficult.

$>$ Inability to communicate the differentiator at a more targeted level. (Long elevator speech.)

$>$ Single accounting system, making managerial information too broad and blended.

$>$ Executive management team must focus on a matrix of six capabilities across three markets and four stage of the product life cycle.

$>$ Could not pace growth at different speeds for unique individual capabilities or markets.

\section{Option B: Reorganize by market segments that more closely aligned with typical competitors.}

Pros

$>$ Sales force and operations could develop a stronger mastery because of the narrower products and solutions.

$>$ Accounting could restructure for more precise and specific measures in each individual newly established profit center.

$>$ Individual performance could be better measured; leadership could better identify training and development opportunities.

$>$ Avoid the risk of "Exhausted Mediocrity".

$>$ Remove the disparity of cultures found between technologists, government contractors, and building construction associates.

\section{Cons}

Higher potential total SG\&A expense.

$>$ Indirect job cost would be funded through smaller pools, necessitating higher indirect rates in the cost model.

$>$ Barriers to performing highly complex large projects, entailing all six current capabilities, with a single project manager.

$>$ Added overhead as three project managers may be needed to represent all the three separate P\&L resource providers.

$>$ Clients having three points of contact for same services, sales, and operations.

$>$ Accounting producing three work products, meaning lost efficiency and higher total cost. 


\section{Option C: Reorganize by product life cycle.}

\section{Pros}

Sales force and operations could develop a stronger mastery because of the narrower requirements, separating small services from large projects.

$>$ Accounting could select vendors that implement industry specific software for the O\&M services for the operations and maintenance organization.

$>$ Accounting could select vendors that implement industry specific software for the project and job cost needs of the design build organization.

$>$ Minimized risk of "Exhausted Mediocrity", and development of a mastery of customer satisfaction for the life cycle specific requirements.

\section{Cons}

$>$ Redundant tooling and equipment expenses driving indirect cost higher.

$>$ Higher potential total SG\&A expense.

$>$ Added overhead to duplicate executive level leadership.

$>$ Clients with two points of contact for same services, sales, and operations.

$>$ Accounting producing two work products, meaning lost efficiency and higher total cost.

\section{Option D: Reduce number of capabilities with a single team.}

\section{Pros}

Sales force and operations could develop a perfect mastery because of the single narrow mission.

$>$ Simplified accounting could select one vendor that implements industry specific software platform.

$>$ Eliminated risk of “Exhausted Mediocrity”, and development of a mastery of narrow technologies and clients.

$>$ Clients enjoying a single point of contact and having a clear understanding of their offering and brand.

\section{Cons}

Higher potential total SG\&A expense.

$>$ Lower sales revenue and a need to grow through market share only.

$>$ No way to compete with large business or differentiate from other high quality small businesses.

$>$ Smaller total market opportunity, little or no diversification from market or industry cycles.

Enough pondering, he thought, it's time to act on these options and choose a strategy for 2015 and forward. Leadership must be decisive; it was time to take a final review of the pros and cons and act. 


\section{References}

Fischer, J. (2006). Navigating the growth curve. Boulder: Growth Curve Press.

Frei, F., \& Morriss, A. (2012). Uncommon service: How to win by putting customers at the core of your business. Boston: Harvard Business Review Press.

Gonzalez, G. T. (2014). Mission Critical Solutions (Strategic Plan \& Business Plan). Tampa, Florida.

Mohr, L. B. (1982). Explaining organizational behavior. San Francisco: Jossey-Bass.

\section{Biography}

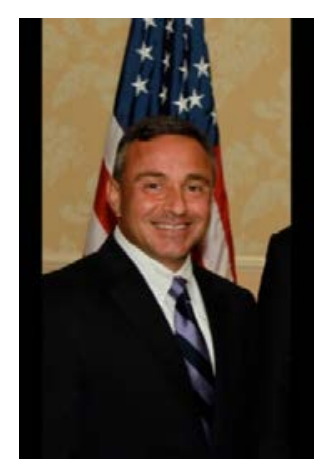

As founder and president of Mission Critical Solutions, Inc., a leading-edge technology solutions provider, Gilbert Gonzalez leads branch and project offices located throughout the United States. His vast experience in designing IPConverged Technology and IT/telecommunications mission critical solutions for commercial, military, and other federal, state and local government clients helped the firm become the fastest-growing privately owned technology firm in Tampa Bay for four consecutive years. The company is a two-time finalist for the Greater Tampa Bay Chamber of Commerce Small Business of the Year. In 2005, Gonzalez was named the Small Business Person of the Year for Florida by the U.S. Small Business Administration. He is a frequent lecturer at business forums and, in addition to his service at MCS; Gonzalez has published software and case studies related to strategic management and business policy. Gonzalez holds both Low-Voltage and Unlimited Electrical Contractor licenses and he is a Registered Communication Distribution Designer. He received an MBA from University of South Florida in Tampa, FL, doing so with honors and with concentrations in management of information systems and economics. 


\section{Exhibit 1: MCS Geographic Service Area}

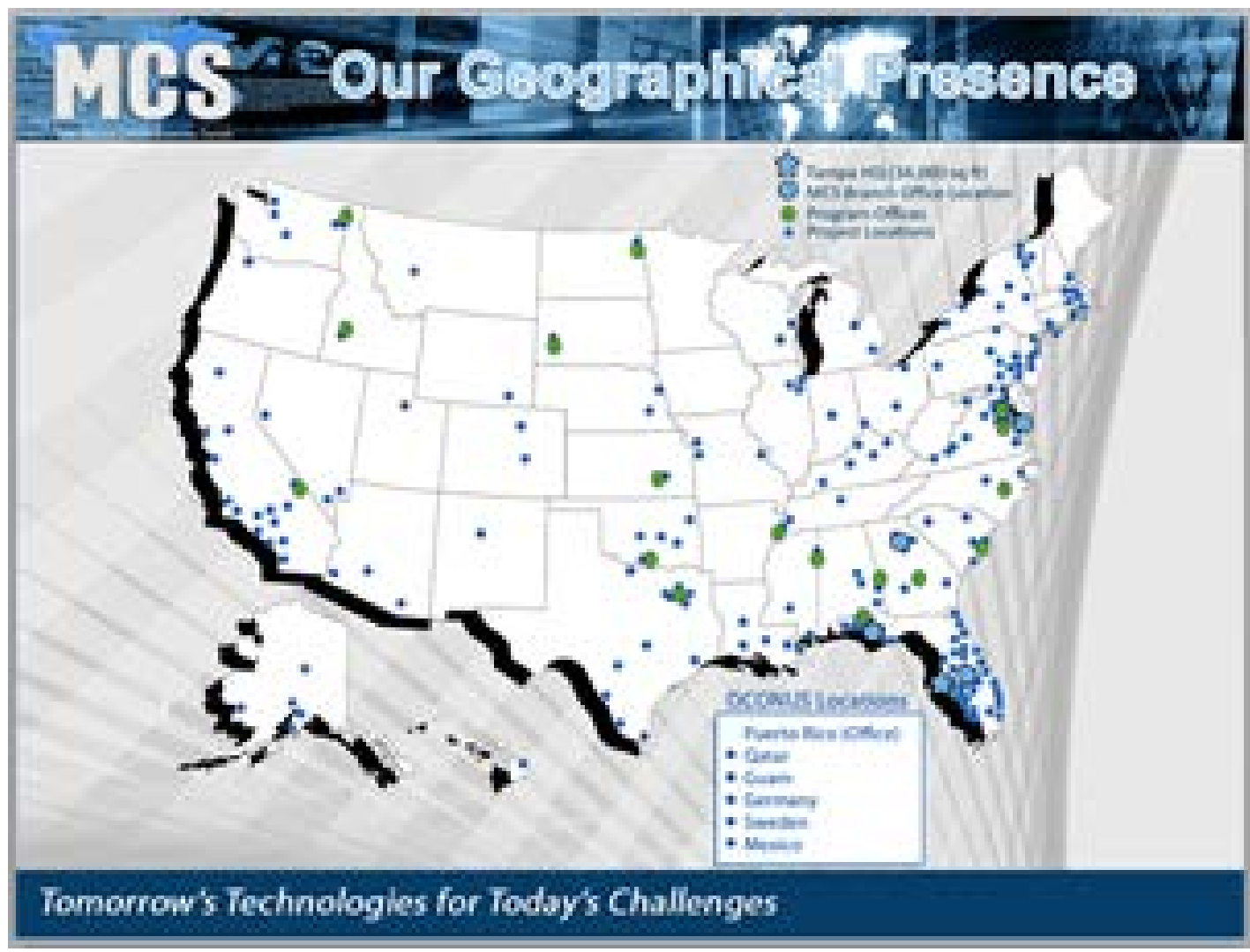

Source: Developed by case writer. 


\section{Exhibit 2: Current Strategic Audit}

\section{Mission Critical Solutions Strategic Audit 2014}

\section{The Current Mission Statement}

The firm's mission is to provide a reliable, competitively priced, and superior mission critical IPConverged Technology solutions to Federal, state, and local governments, educational institutions, and commercial markets primarily in the continental United States (CONUS) and strategically outside the continental Unites States (OCONUS), as well as in forward support of Department of Defense (DOD) client initiatives within our six categories of capabilities.

\section{The Current Objectives}

To increase shareholder return in a risk-balanced manner then ensures organizational continuity beyond the founder's career.

To grow our revenues and simultaneously rebalance our revenues across the Federal, SLED, and commercial market places.

To align objectives of all internal and external stake holders where possible.

To maintain a lean SG\&A exposure and leverage EBIDA by creating volume through the efficiencies of six capabilities serving three markets with a single general and administrative cost center.

To provide a timely and objective measurement of performance to each associate, supplier, and subcontractor, in order to encourage and enhance organizational priorities.

To evolve the organizational structure, business procedures, and practices in order to empower middle management team to maintain and improve quality, customer satisfaction, associate satisfaction, and shareholder return through efficient cost strategies.

\section{The Current Strategies}

MCS shall maintain profitable growth in revenue and profits through horizontal and vertical marketing strategies by simultaneous expansion of capabilities, clients, and geographic markets.

MCS shall provide key monetary and non-monetary metric reporting to the field in a real time fashion with standardized reporting, dashboard portals, and automated alerts with an escalation protocol.

MCS shall expand our business relationship with converged technologies manufacturers and complimentary industry service providers.

MCS shall implement adoption and use of state of the art technologies for accounting and collaboration through cloud, UC, and thin client technology.

MCS shall continue to develop core capabilities that differentiate through the acquisition of new engineering skills and the continued development of the current sales and operational engineering team. 
Examples include new emerging converged security, surveillance, communications, and multimedia products to integrate into our clients IP networks and enhance the support of their mission.

MCS shall expand our customer base and market share, through remote workforce integrated with state of the art collaboration technology, and establish new operational programs throughout the continental United States (CONUS).

MCS shall market for long-term operations and maintenance programs, IT, telecommunications, and other converged IP systems programs through Federal sales targeting key contract vehicles.

MCS shall continue to expand and invest in its capture and proposal capabilities for our Federal and DOD markets.

MCS shall expand our capabilities via constant recruiting of specialized engineering, project management, and business development skills via our recruiting department. Particular emphasis is placed on DOD cleared personnel.

MCS will review and update our IT infrastructure to integrate our UC, mobile strategies, CRM, project management, sales engineering, and back office processes, with emphasis on training staff and integrated deployment of the tools over acquisition and availability, that goes underutilized or not adopted.

MCS will review and update our incentive and bonus structure to establish equitable performance incentive systems that reward exemplary individual performance and align the individual goals with that of the team (corporation).

MCS will implement top to bottom communication and information flow in order to motivate, inform, and empower all team members from entry level roles to top management.

MCS will require every employee to demonstrate their contribution through measurable metrics, reviewed on appropriate time intervals. Coaching and training will be linked to these reviews

MCS will improve communications and encourage collaboration throughout the organization. MCS shall establish a protocol for effective communications and resolve frustrations quickly in a constructive manner.

\section{Internal Analysis}

\section{MCS's current policies}

Mission Critical Solutions shall provide high availability and reliable services and products with a passionate and motivated team of associates in a stimulating and progressive culture. Standards of honesty and ethics in all dealings with all stakeholders shall continue to be at the highest bar achievable. Employees shall be empowered to succeed. All policies and procedures shall be overridden when they fail the test of ethics or common sense, as it relates to the clients desired result.

\section{Corporate Culture}

MCS's culture is a driven, innovative and solutions-oriented environment. MCS operates with the understanding that effort is not a substitute for results for the client. We will continue to provide a high energy, challenging work environment. MCS's path will remain on the ethical high road. MCS's team 
will maintain an insatiable, passionate, results driven desire to succeed. MCS's culture will continue to encourage a team atmosphere and camaraderie among the associates. MCS's management means and methods will always align the associates, the client, and the shareholders goals and objectives.

\section{Top Management}

MCS's senior management team is a highly qualified and proven group with combined education and experience necessary to continue our growth and industry-beating margin performance. The same proven team that led us through the worst economy in two decades remains intact. It now accepts the challenge of leading our branches and programs via our talent rich middle level managers into this next period of growth. Management shall continue to delegate authority and empower our team closest to the client and the work. Through evaluation and control, we will assure the high quality performance of their subordinate team. Top management will continue to lead by example, demonstrating our culture and values to the current and future associates.

\section{MCS's Sales and Marketing}

\section{Strengths}

MCS has an established and experienced inside and outside business development team that has the ability to identify and target end users, Federal, state and local, as well as commercial, who are interested in and in need of our capabilities.

MCS has several online services that are highly effective in identifying new customers and opportunities in the Federal, state, and commercial markets.

Federal trade shows and networking via organizations such as AFCEA continue to be effective ways of marketing and branding our company.

MCS has a large portfolio of contract vehicles both as prime and through teaming agreements. This allows our Federal and affiliated state, local, and education customers to procure our solutions without further competition.

MCS strategy of targeting clients who are in need of regular and reoccurring services and goods sustains our sales and profits and creates incremental growth with each new customer.

MCS has established staff and programs in twelve states. This creates the opportunity to expand our commercial services and products where a geographic presence is necessary.

MCS's metrics based management model allows us to effectively reward the best and brightest while quickly identifying associates unable to perform.

MCS's bonus and commission system encourages the best of class within our industry to seek employment with our firm.

We market to the new construction market place through the bid rooms and telemarketing. This identifies building automation, core infrastructure opportunities, and future operations and management service clients.

MCS's product lines are designed to include the full life cycle from design and implementation through support and maintenance. This gives every new relationship the potential to become a revenue and profit annuity. 
MCS has an established Federal proposal team capable of presenting our solutions and capabilities to the market place via oral and written proposals, with win rates in excess of $50 \%$.

MCS's ability to attract and court vendors and teaming partners is at an all-time high because of our Federal knowledge and winning track record.

\section{Weaknesses}

MCS has been unable to recruit enough top $1 \%$ of sales professionals outside of the federal space. This impedes growth in SLED and commercial markets.

While marketing for subcontracts and vehicles have been successful. Numerous disappointments with limited competition contracts have been the trend since the "Fiscal Cliff and Sequestration (The Federal recession). Funding removed from winning efforts has been a challenge.

MCS does not have permanent presence in D.C. and the belt way.

No longer the commercial price leader, recessionary margins have commercial markets operating at margins that are breakeven or short-term losses. There are some signs of recovery.

MCS lacks clear and timely reporting and still uses subjective tools for evaluation and control of sales performance in spite of the Salesforce CRM.

Sales associates are not qualified in all six capabilities, and they may feel overwhelmed.

\section{Sales Engineering Services}

\section{Strengths}

MCS has a deep bench of certified engineers, designers, and estimators. The team holds the following certifications: RCDDs, MCSE, CCNAs, NICET, numerous Avaya design and installation, Master Electricians, Cisco, NEC, CTIs, Crestron, AMX, Avigilon, Pelco, and numerous SCS certifications. Many more not listed.

Sales engineering billed in excess of 900K last year, covering a significant part of their overhead.

Automated design tools, estimating solutions, and CAD capability are increasing quality and efficiency of the team.

MCS's Sales and Engineering team is highly visible to our client. Clients often build relationships with the team and express a desire to work with MCS to stay collaborative with our team in Sales

Engineering.

\section{Weaknesses}

MCS's estimating model was less competitive recently due to tinkering with productivity goals and historically high indirect rates. Indirect rates are negatively impacted by the reduced volume of labor hours and increasing cost of fringe benefits for our workers. 
MCS engineers must balance their time between estimating, quality control, and operational support. Prequalifications are not always thorough, resulting in wasted time and lost bids.

\section{Accounting and Finance}

\section{Strengths}

The strong team is led by an experienced CFO and highly capable Controller. External auditors and current CPAs are exceptional at getting MCS through the flurry of unforeseen volume of third party audits.

MCS had a large increase in assets and equity for ' 14 , mainly due to onetime real-estate transactions created by the recession.

MCS has ample working capital with lines in excess of 8 million dollars and near zero short-term rates.

MCS established a fixed rate mortgage on headquarters properties to ensure a low cost of capital for current facility in perpetuity. Further, MCS has ample room to grow within the new facility.

MCS has a long relationship with a great bank that provides solid services and advice.

\section{Weaknesses}

Payroll management still not clean; Davis Bacon, certified payrolls, and service contract act requirements across 12 states are challenging for our accounting team.

Inability to separate cost control from cost minimization mentality; employees want a fixed process for procurement. We lack experienced negotiating skills at the day-to-day procurement level.

MCS is dependent on large jobs to create volume to offset smaller margins and sustain profitability in the near future.

Industry margins slow to return, it is still a buyer's market with respect to price.

\section{Operations and Programs}

\section{Strengths}

MCS has built team depth and experience in all six capabilities; we anticipate further benefits from cross training key personnel.

MCS has the specialty tooling needed to create a competitive advantage over our competitors.

MCS staffs Project Management Professionals (PMPs) with broad operational experience among the key project managers. Integration of the six capabilities necessitates PMP disciplines be practiced.

MCS's operational workforce accepts difficult schedule and productivity challenges in a positive manner.

MCS has a deep benched of secret and top-secret cleared associates available at will or outsourced for a longer duration, if needed. 


\section{Weaknesses}

MCS's employee morale must be monitored. Challenging integrations, schedule acceleration, and problem solving under duress can take their toll on our team.

MCS’s middle managers express frustration over the difference between cost controls and cost minimization.

MCS can have difficulty in recruiting the primary skilled workers at budgeted cost.

MCS has experienced difficulty in managing the fringe benefit pool for our project-based employees, whose duration of employment can be less than six months.

MCS's traditional IT services labor market is oversupplied, with demand for external service providers falling.

\section{Human Resources and Recruiting}

\section{Strengths}

Many new talented staff members have been added due to recent growth.

Protected employee program is retaining key personnel.

MCS has two full time recruiters, a senior and junior recruiter. The senior recruiter is resourceful, talented, and innovative. They do a valiant job supporting both proposal and day-to-day staffing needs.

Using state of the art social media tools, we are able to reach far and wide to find qualified new associates.

\section{Weaknesses}

Tight economy has placed pressure on wage reviews and enhancements.

Increased overhead and fringe cost year over year continue to outpace inflation.

Limited availability of specialized engineers and technicians such as Avaya and audio video makes building our own necessary.

Cost of certifications, training, and skills enhancement is difficult to control. Training for MCS is still ad hoc or third party; we do not have a formal strategy.

Our teams have developed a "look the other way" behavior when a peer is performing below standard or resources are being wasted. Many employees prioritize their peer relationships over their responsibility to the shareholder. MCS needs to establish the Elie Wiesel mantra into its value system: "To be indifferent and silent is the greatest sin of all". 


\section{External Analysis (opportunities and threats)}

\section{Opportunities}

Emergence of low voltage security systems converging onto the network continues; endpoints from every system are now the norm.

VOIP and multimedia technology have blurred the line between telecommunications, $\mathrm{A} / \mathrm{V}$, and IT. Unified communications is under heavy adoption.

Building automation is now integrated in the new construction and renovation markets making general contractors our newest points of contact.

Outsourcing opportunities in the Federal market place are growing again, but margins are thin and large businesses' ravenous appetites makes this market competition more difficult than any other time.

Power cell and energy management revolution and green design are influencing our systems, especially C4 within the DOD space.

Economic recovery combined with low interest rates sets the stage for new growth; we must be quick to respond.

UC is on this rise, IP Surveillance is displacing legacy systems, wireless networks are moving into building infrastructure, fire alarm is on the network, $\mathrm{A} / \mathrm{V}$ is an app, and monitors and speakers are an output device on the network. Distributed digital signage now replaces legacy signage, technology and marketing blend to create a niche, and convergence is growing at an increasing rate. Email is a notification tool on all endpoints and devices are in automation. The cloud untethers workers.

Wireless bandwidth redefines the edge of the network.

\section{Threats}

Electrical contractors' market place controls our ability to offer a solution to our customer directly in the initial design stages.

Wireless technology is reducing the dollar value of the SCS opportunity.

As other markets converge, new competition enters into our spaces as well.

Lack of capital in the technology market has slowed new product development and lengthens the ownership time of equipment.

Clients are deferring CAPEX and looking to lower OPEX still; the recovery happened and no one noticed. It was too gentle to reestablish an aggressive growth and investment strategy. Stock buy backs displace CAPEX spending in all areas. IT spending is seen as reoccurring OPEX and evaluated very stringently.

Cloud computing and virtualization are examples of shrinking cost to our clients, which are shrinking revenues to our industry.

We must obsolete ourselves with solutions that meet the markets goals. All new products are tomorrow's commodities. Customers will separate the product from the labor when procuring solutions. 


\section{Supplier and Vendor Analysis}

\section{Opportunities}

Current partners parallel our growth plans into newly converged technologies.

MCS is perceived as a top tier account. Both manufacturers and distributors invest time and money in our partnership via training and collaborative sales.

\section{Threats}

Some vendors route work to less sophisticated competitors, who buy inefficiently, because they enjoy higher profit margins with smaller, more relaxed buyers.

Channel conflict often causes stress when vendors specify MCS out of an opportunity.

There is ample credit in the marketplace, but stringent shorter duration terms still prevail. This places increased pressure on MCS's working capital.

\section{Key Strategic Factors}

MCS has a high cost of sales and a low sales efficiency ratio (revenue / sales expense) relative to its more narrow competitors. Marketing efficiency in both commercial and Federal markets must improve through better prequalification, proposal creation, and closing techniques. Improving the close ratio will drive growth and improve our sales efficiency ratio.

MCS's corporate culture is becoming diluted and varies by team and region. The impact of functional teams spread across 22 states has diluted the driven and competitive culture found in the Tampa office. We must improve communication and foster a single culture across the geographically dispersed teams.

MCS's current performance appraisal system is ill-supported by blended metrics which allow poor and moderate performers to hide. Further, it makes mentoring and coaching more challenging due to the lack of objective data available per individual. The team approach needs to be balanced with individual performance metrics.

MCS's financial model depends on driving enough volume to fund the indirect cost pools needed. When revenue volume falls our cost, fixed indirect cost are not funded and profits suffer. We must emphasize sales volume in order to overfund indirect pool and outperform the market on return.

MCS can take on highly complex projects and deliver a converged solution in less time and at lower cost than its larger business rivals.

Source: Developed by case writer. 


\section{Exhibit 3 Current Organizational Chart}

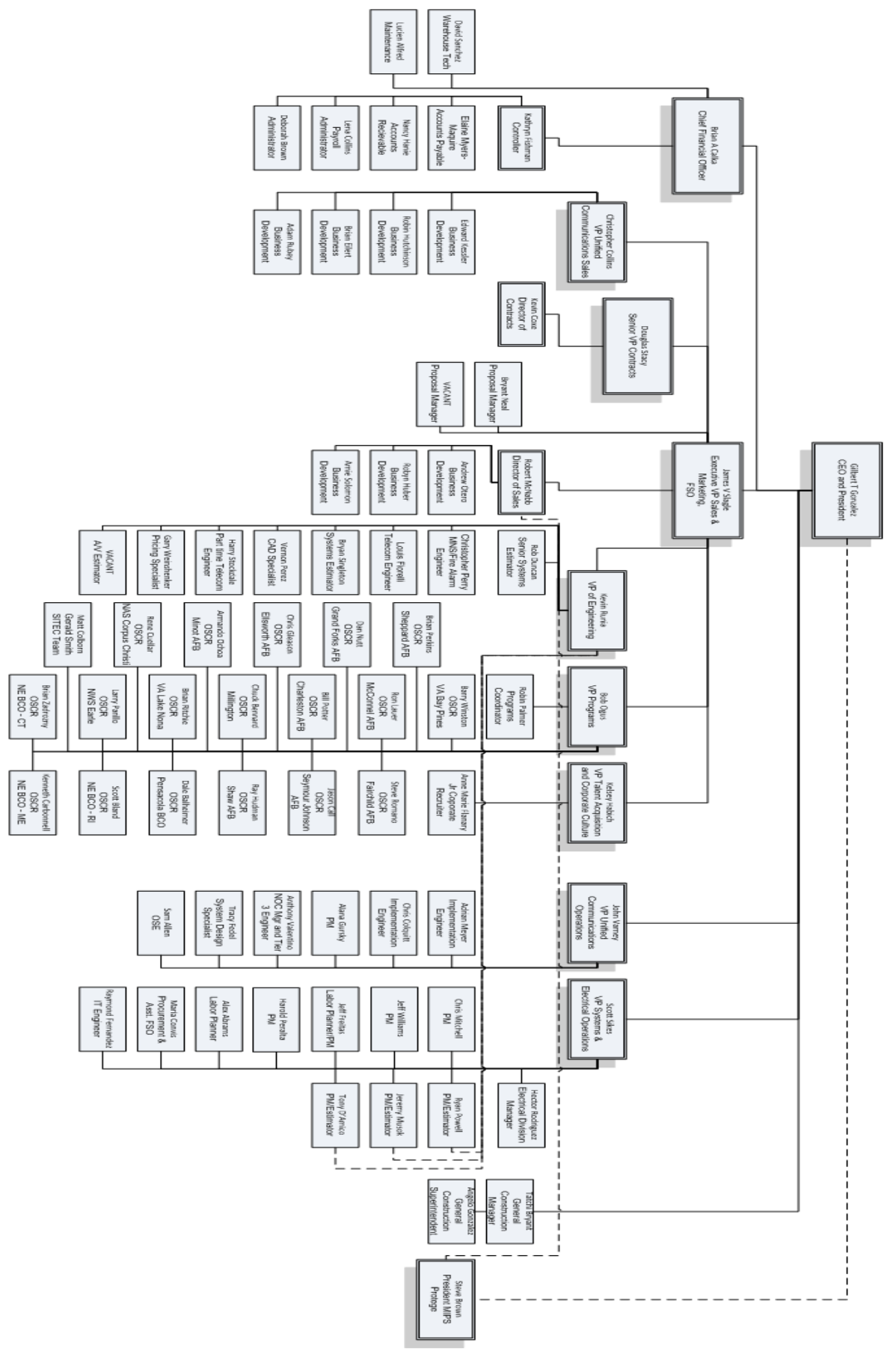




\section{Exhibit 4: Capabilities}
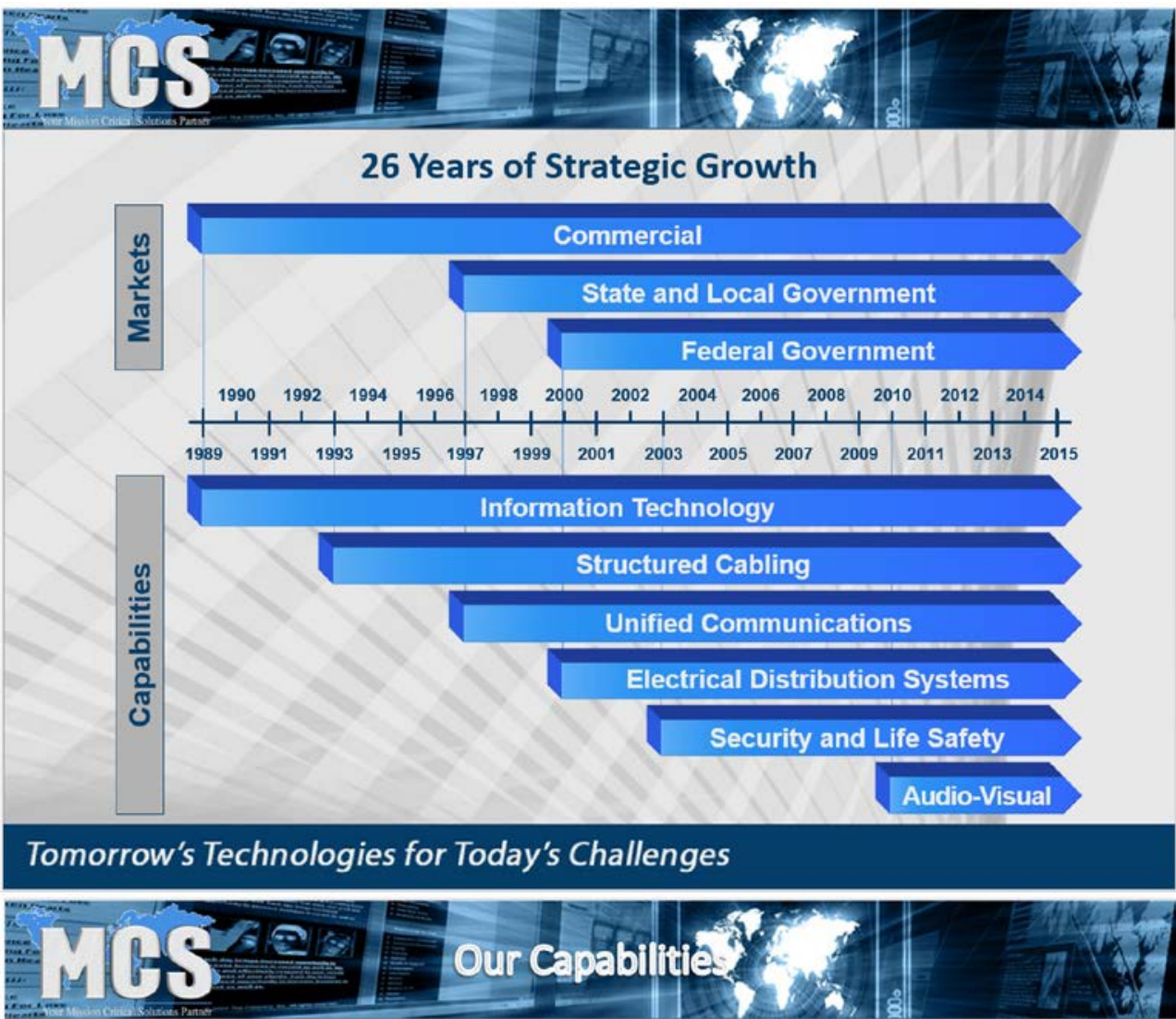

- Data, Voice, A/V and SIP

structured cabling

- RCDD Cabling Design

Assistance

- Inside and Outside

Fiber \& Copper

Implementation
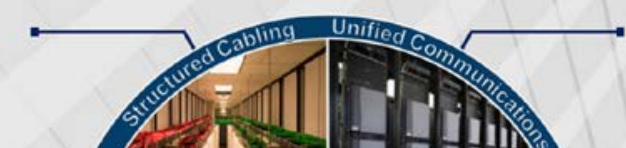

Voice over Interne Protocol (VolP)

- Broadband Wireless

- Video Teleconferencin

- Enterprise PBX Design

- System Administration

- Performance

Monitoring

Consolidate Data

Centers

- Tower Construction

- Design-Build

- Industrial Lightin

- Solar Power

- UPS \& Generators

- Utility Plants

- Facility Buildout

- BIM 3D Modeling

LEED Construction

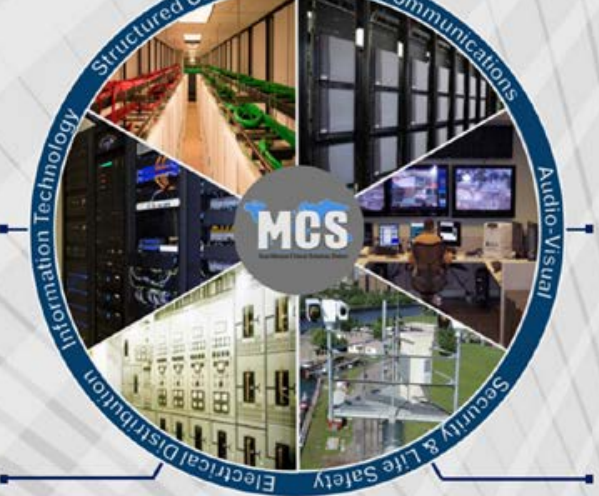

Tomorrow's Technologies for Today's Challenges

Source: Slagle, J. (2014). Mission Critical Solutions--2014 Capabilities. 